\title{
The Exponentially Increasing Rate of Patients Infected with COVID-19 in Iran
}

\author{
Leila Moftakhar, MSc'; Mozhgan Seif, $\mathrm{PhD}^{2 *}$ \\ ${ }^{1}$ Student Research Committee, Shiraz University of Medical Sciences, Shiraz, Iran \\ ${ }^{2}$ Department of Epidemiology, School of Health, Shiraz University of Medical Sciences, Shiraz, Iran
}

\begin{abstract}
Background: Coronavirus, the cause of severe acute respiratory syndrome (COVID-19), is rapidly spreading around the world. Since the number of corona positive patients is increasing sharply in Iran, this study aimed to forecast the number of newly infected patients in the coming days in Iran.

Methods: The data used in this study were obtained from daily reports of the Iranian Ministry of Health and the datasets provided by the Johns Hopkins University including the number of new infected cases from February 19, 2020 to March 21, 2020. The autoregressive integrated moving average (ARIMA) model was applied to predict the number of patients during the next thirty days. Results: The ARIMA model forecasted an exponential increase in the number of newly detected patients. The result of this study also show that if the spreading pattern continues the same as before, the number of daily new cases would be 3574 by April 20 . Conclusion: Since this disease is highly contagious, health politicians need to make decisions to prevent its spread; otherwise, even the most advanced and capable health care systems would face problems for treating all infected patients and a substantial number of deaths will become inevitable.

Keywords: COVID19, Forecast, Iran

Cite this article as: Moftakhar L, Seif M. The exponentially increasing rate of patients infected with COVID-19 in Iran. Arch Iran Med. 2020;23(4):235-238. doi: 10.34172/aim.2020.03.
\end{abstract}

Received: March 25, 2020, Accepted: March 27, 2020, ePublished: April 1, 2020

\section{Introduction}

Coronaviruses are a large family of enveloped viruses with single-stranded RNA and a crown on their surface. ${ }^{1,2}$ These viruses are severely pathogenic for humans, causing respiratory infection, liver disease, and gastrointestinal and neurological diseases. ${ }^{3}$ Two outbreaks of coronaviruses, SARS-CoV-1 and MERS-CoV, occurred in 2003 and 2012, respectively. ${ }^{3}$

The novel coronavirus was reported in December 2019 with the emergence of several cases of pneumonia of unknown etiology that caused severe acute respiratory syndrome in Wuhan city, China.-8 Some of these cases were seen in people who used seafood in Wuhan. ${ }^{3,9}$

The novel coronavirus was introduced as coronavirus disease 2019 (COVID-19) by the World Health Organization, that probably originated from bats. ${ }^{4}$ However, its origin has not been confirmed yet. ${ }^{10,11}$ COVID-19 is an infectious disease caused by SARSCoV-2. ${ }^{6}$ Symptoms include fever, cough and shortness of breath $^{10}$ The disease is transmitted through inhalation of respiratory droplets, close contact with the infected person, and contact with contaminated surfaces or objects. ${ }^{3}$ The incubation period of COVID-19 is over 14 days and during this period, the virus can be transferred to other people. $^{3}$

COVID-19 spread rapidly in China ${ }^{6,12}$ and around the world, ${ }^{7}$ and was introduced as a pandemic by the WHO on March 11, 2020. ${ }^{13}$ By March 22, the number of infected countries was more than 180 and the number of confirmed patients and deaths were 314135 and 13560 people in in the world, respectively. ${ }^{14}$ Although the disease is controlled in China, it is increasing in other countries of the world including Iran. ${ }^{15}$ Iran experienced the first case of COVID-19 in Qom on February 19, 2020. Then, the disease spread rapidly throughout the country. ${ }^{16}$ As of March 22, 2020, the total number of confirmed patients has been $21638 . .^{14}$

Some of the most important questions are when the spread of COVID-19 will end, what the trend of this outbreak looks like, and how many people would be infected with COVID19 daily. These are the main concerns of people and health policy makers. These questions could be answered through predictive models. ${ }^{2,6}$ Therefore, due to the increasing rate of coronavirus positive patients in Iran, this study was conducted to forecast daily new infected cases for next days in this country.

\section{Materials and Methods}

This time-series study was undertaken to forecast the number of new cases of COVID-19 in Iran, until April 20, 2020. The data used in this study included the number of new cases from February 19 to March 21, 2020 in 
Iran. New cases are all cases that have been approved by laboratory tests. Data were extracted from two sources. First: the daily reports of the Iranian Ministry of Health and Medical Education, which included the number of new cases, new remissions and new deaths. Second: open datasets provided by the Johns Hopkins University, which provides information cumulatively for the general public.

\section{Statistical Model Building}

The autoregressive integrated moving average (ARIMA) model was used for forecasting time-series data in order to forecast the number of newly infected patients. ARIMA ( $\mathrm{p}, \mathrm{d}, \mathrm{q}$ ) is actually simultaneous fit of other two models including Auto Regressive (p) and Moving Average (q) ${ }^{17}$ The plot of residuals was used in addition to Autocorrelation and Partial Autocorrelation Functions (ACF \& PACF) to assess the model's goodness of fit. Residuals were also tested to be stationary using Box-Ljung.

Box-cox is another popular transformation to provide stationary time-series. ${ }^{18}$ It should be noted that BoxCox transformation was applied to prepare data for fitting $\operatorname{ARIMA}(0,1,0)$, by use of 'forecast' package in $\mathrm{R}$ software. Statistical significance was set at 0.05 and the model goodness of fit was assessed through inspection of residuals.

\section{Results}

The observed trend of new cases from February 18 to March 21, 2020 is displayed in Figure 1. This Figure also shows the forecasted number for thirty days ahead, by ARIMA model (Table 1). Obviously, an exponential increase is clear in the daily number of newly detected patients. According to this prediction, if the spreading pattern continues similarly to the observed pattern, the number of daily new cases would be 3574 by April 20 .

The plot of residuals versus observations' order showed no pattern. It seems that they were randomly scattered around zero (Figure 2a). Autocorrelation and Partial Autocorrelation Functions showed no spike and this implied that there was no remaining auto coloration regarding the residuals (Figure 2). The Shapiro-Wilk test was used to check the normality of residuals ( $P$ value $=0.60$ ), in addition to Normal Probability Plot and Histogram of residuals which did not show any substantial deviation from normality (Figure 3). All residual assessment confirmed goodness of fit for the fitted ARIMA model, as depicted in Figure 1.

\section{Discussion}

This study was conducted to forecast the number of the daily new cases infected with COVID-19 until April 20, 2020 in Iran using the ARIMA predicting model. The total number of confirmed patients and deaths in Iran was 21638 and 1685, respectively, until March 22, 2020. The results of our study indicate that if the spreading pattern continues as before, there will be a sharp increase in the number of new cases in the next days. Based on our predictions, the number of new cases would be 3574 on April 20, 2020 according to ARIMA.

Of course, the accuracy of this prediction depends on the accuracy of applied data and the adequacy of the applied statistical model. Even if the forecast is overestimated and only a fraction of this prediction is realized, Iran's health care system would face an extremely difficult problem. Given that the disease is transmitted through respiratory droplets and is spreading rapidly, this forecast is important for health planning.

Finally, we must say that we do not have additional evidence that can estimate the exact number of patients, but we hope that our results could help timely decisions by health policy makers in Iran in providing adequate hospital equipment, medical and nursing staff and essentials needs, in order to prevent a more serious crisis.

\section{Limitations}

Two major limitations should be considered in this study. First, due to the lack of data at the individual level, including patients' demographic information, their social networks

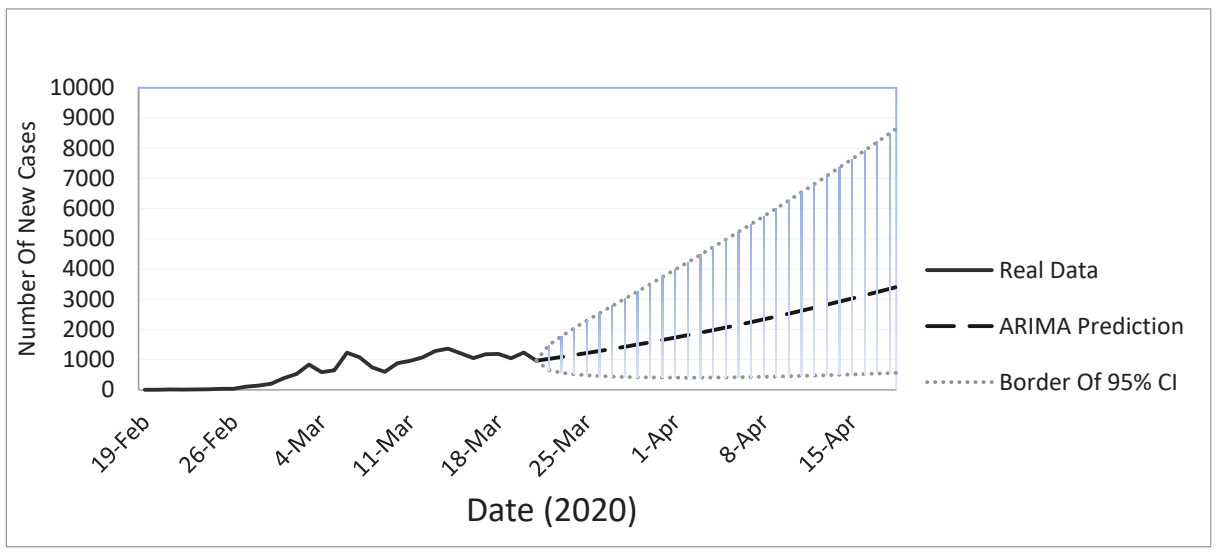

Figure 1. Forecasted and 95\% Confidence Interval of New Cases with Covid19 in Iran; until April 20, 2020. 
Table 1. Forecasted Number of Daily New Cases with 95\% Confidence Intervals

\begin{tabular}{|c|c|c|c|}
\hline \multirow{3}{*}{$\begin{array}{l}\text { Days } \\
\text { (of 2020) }\end{array}$} & \multicolumn{3}{|c|}{ Prediction } \\
\hline & \multirow{2}{*}{ ARIMA } & \multicolumn{2}{|c|}{$95 \% \mathrm{CI}$ for ARIMA } \\
\hline & & Lower & Upper \\
\hline 22-Mar & 1026.402612 & 653.1516367 & 1483.652205 \\
\hline 23-Mar & 1088.63684 & 567.8413155 & 1777.429597 \\
\hline 24-Mar & 1152.702682 & 516.4981522 & 2040.903061 \\
\hline 25-Mar & 1218.600139 & 481.6742792 & 2291.520464 \\
\hline 26-Mar & 1286.329211 & 456.8553398 & 2535.796164 \\
\hline 27-Mar & 1355.889898 & 438.8200933 & 2776.951401 \\
\hline 28-Mar & 1427.2822 & 425.7248814 & 3016.829833 \\
\hline 29-Mar & 1500.506117 & 416.4136128 & 3256.587551 \\
\hline 30-Mar & 1575.561648 & 410.1144707 & 3496.996372 \\
\hline 31-Mar & 1652.448795 & 406.2881487 & 3738.595604 \\
\hline 1-Apr & 1731.167556 & 404.5445755 & 3981.775316 \\
\hline 2-Apr & 1811.717933 & 404.5939391 & 4226.825321 \\
\hline 3-Apr & 1894.099924 & 406.2162804 & 4473.965578 \\
\hline 4-Apr & 1978.31353 & 409.2417741 & 4723.365913 \\
\hline 5-Apr & 2064.358751 & 413.5374742 & 4975.159271 \\
\hline 6-Apr & 2152.235587 & 418.9981334 & 5229.4509 \\
\hline 7-Apr & 2241.944038 & 425.5396791 & 5486.324872 \\
\hline 8-Apr & 2333.484103 & 433.0944766 & 5745.848822 \\
\hline 9-Apr & 2426.855784 & 441.6078204 & 6008.077456 \\
\hline 10-Apr & 2522.059079 & 451.0352951 & 6273.055188 \\
\hline 11-Apr & 2619.09399 & 461.3407578 & 6540.818162 \\
\hline 12-Apr & 2717.960515 & 472.4947767 & 6811.39581 \\
\hline 13-Apr & 2818.658655 & 484.4734088 & 7084.812075 \\
\hline 14-Apr & 2921.18841 & 497.2572311 & 7361.086379 \\
\hline 15-Apr & 3025.54978 & 510.8305679 & 7640.234398 \\
\hline 16-Apr & 3131.742765 & 525.1808665 & 7922.268685 \\
\hline 17-Apr & 3239.767364 & 540.2981911 & 8207.199176 \\
\hline 18-Apr & 3349.623579 & 556.1748077 & 8495.033605 \\
\hline 19-Apr & 3461.311409 & 572.804843 & 8785.777845 \\
\hline 20-Apr & 3574.830853 & 590.1840004 & 9079.436192 \\
\hline
\end{tabular}

and travels, no risk factor for this disease was assessed and studied. Second, the small number of observations for this type of time-series algorithms is another major limitation of this study as models might not be trained very well. However, the prediction of this study may be useful for health decision makers; therefore, it was not reasonable to waste time for data provision.

\section{Conclusion}

In conclusion, the result of this study is an alarm for health policy planners and decision makers to make timely decisions regarding the supply of essential equipment for hospitals.

\section{Authors' Contribution}

ML searched and provided data, wrote, edited and critically reviewed manuscript. SM designed the study, performed statistical analysis and wrote the manuscript. The final version of the manuscript was approved by all authors.

\section{Conflict of Interest Disclosures}

None. (a) ARIMA $(0,1,0)$ Residuals Versus Observatio Order

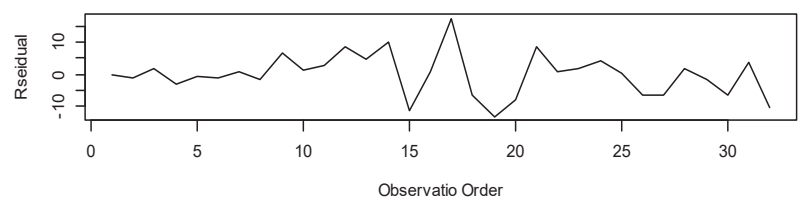

(b) ACF of ARIMA $(0,1,0)$ Residuals

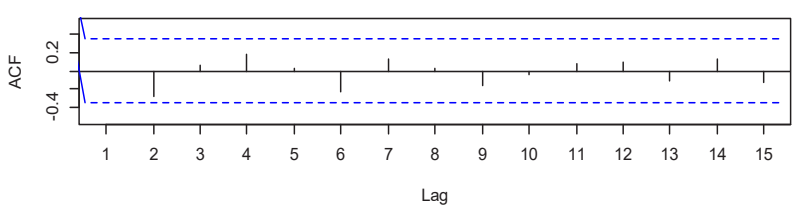

(c) PACF of ARIMA $(0,1,0)$ Residuals

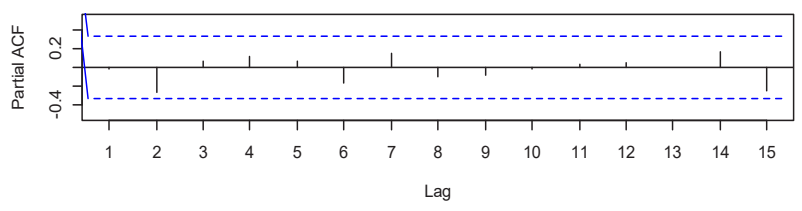

Figure 2. Residual Assessment of ARIMA Model Including (a) Plot of Residuals Versus Observation Order, (b) Auto Corrolation Function of Residuals, and (c) Partial Auto Corrolation Function of Residuals.

(a) Normal Probability Plot of ARIMA $(0,1,0)$ Residuals

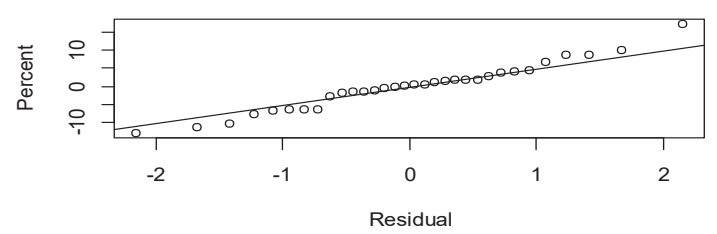

(b) Histogram of ARIMA $(0,1,0)$ Residuals

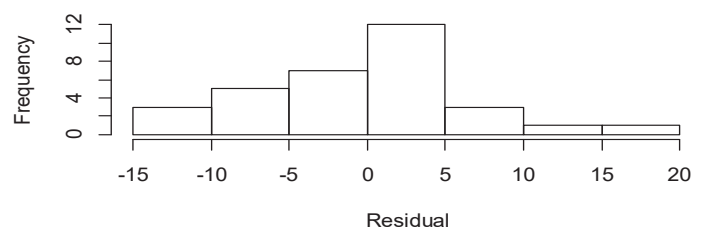

Figure 3. Residual Assessment of ARIMA Model Including (a) Normal Probability of Plot and (b) Histogram of Residuals.

\section{Ethical Statement}

In this study we applied the information about the number of new cases with Covid1 9 in Iran. The data was provided by Johns Hopkins University and Iranian Ministry of Health. Therefore it seems that ethical approval is not required.

\section{References}

1. Wan H, Cui JA, Yang GJ. Risk estimation and prediction by modeling the transmission of the novel coronavirus (COVID-19) in mainland China excluding Hubei province. medRxiv. 2020. doi.org/10.1101/2020.03.01.20029629

2. Zhang KK, Xie L, Lawless L, Zhou H, Gao G, Xue C. Characterizing the transmission and identifying the control strategy for COVID-19 through epidemiological modeling. medRxiv. 2020. doi.org/10.1101/2020.02.24.20026773

3. Al-qaness MA, Ewees AA, Fan H, Abd El Aziz M. Optimization 
Method for Forecasting Confirmed Cases of COVID-19 in China. J Clin Med . 2020;9(3):674. doi:10.3390/jcm9030674

4. Tang K, Huang Y, Chen M. Novel Coronavirus 2019 (Covid-19) epidemic scale estimation: topological networkbased infection dynamic model. medRxiv. 2020. doi. org/10.1101/2020.02.20.20023572

5. McCall B. COVID-19 and artificial intelligence: protecting health-care workers and curbing the spread. Lancet. February 20, 2020. doi: 10.1016/S2589-7500(20)30054-6

6. Song PX, Wang L, Zhou Y, He J, Zhu B, Wang F, et al. An epidemiological forecast model and software assessing interventions on COVID-19 epidemic in China. medRxiv. March 3, 2020. doi: 10.1101/2020.02.29.20029421

7. Nishiura H, Linton NM, Akhmetzhanov AR. Serial interval of novel coronavirus (COVID-19) infections. Int J Infect Dis. 2020. doi: 10.1016/j.ijid.2020.02.060

8. $\mathrm{Hu} Z, \mathrm{Ge} \mathrm{Q}$, Jin L, Xiong M. Artificial intelligence forecasting of covid-19 in china. arXiv preprint arXiv:200207112. 2020. Available from: https://arxiv.org/abs/2002.07112.

9. Lin Q, Zhao S, Gao D, Lou Y, Yang S, Musa SS, et al. A conceptual model for the outbreak of Coronavirus disease 2019 (COVID-19) in Wuhan, China with individual reaction and governmental action. International Journal of Infectious Diseases. March 04, 2020. doi: 10.1016/j.ijid.2020.02.058

10. Anastassopoulou C, Russo L, Tsakris A, Siettos C. Data-Based Analysis, Modelling and Forecasting of the novel Coronavirus (2019-nCoV) outbreak. medRxiv. March 12, 2020. doi:
10.1101/2020.02.11.20022186.

11. Du Z, Xu X, Wu Y, Wang L, Cowling BJ, Meyers LA. The serial interval of COVID-19 from publicly reported confirmed cases. medRxiv. March 20, 2020. doi: 10.1101/2020.02.19.20025452.

12. Sun K, Chen J, Viboud C. Early epidemiological analysis of the coronavirus disease 2019 outbreak based on crowdsourced data: a population-level observational study. Lancet Digital Health. 2020. doi 10.1016/S2589-7500(20)30026-1

13. Organization WH. Novel coronavirus(2019-nCoV); 2020; Available from: https:/www.who.int/emergencies/diseases/ novel-coronavirus-2019/situation-reports.

14. 14.coronavirus.Report coronavirus cases.2020.Available from: URL: https://www.worldometers.info/coronavirus/

15. 15.Remuzzi A RG. COVID-19 and Italy: what next? Lancet. 2020. doi: 10.1016/S0140-6736(20)30627-9

16. 16.Muniz-Rodriguez K, Fung IC-H, Ferdosi SR, Ofori SK, Lee Y, Tariq A, et al. Transmission potential of COVID-19 in Iran. medRxiv. 2020. doi:10.1101/2020.03.08.20030643

17. Benvenuto D, Giovanetti M, Vassallo L, Angeletti S, Ciccozzi M. Application of the ARIMA model on the COVID-2019 epidemic dataset. Data Brief. 2020;29:105340. doi: 10.1016/j. dib.2020.105340

18. Ansley C, Spivey W, Wrobleski W. A class of transformations for Box-Jenkins seasonal models. J R Stat Soc Ser C Appl Stat. 1977;26(2):173-8. doi: 10.2307/2347025 